\title{
FLUORESCENCE BANDING TECHNIQUES IN THE IDENTI- FICATION OF SIBLING SPECIES OF THE ANOPHELES GAMBIAE COMPLEX
}

\author{
M. GATTI,* G. SANTINI,* S. PIMPINELLI* and M. COLUZZI† \\ * Centro di Genetica Evolutiva del C.N.R., Istituto di Genetica, Università di Roma, Rome, Italy \\ † Istituto di Parassitologia, Università di Roma, Rome, Italy
}

Received 23.ix.76

\section{SUMmaRy}

\begin{abstract}
The mitotic chromosomes of the sibling species $A$ and $B$ of the Anopheles gambiae complex were stained with Hoechst 33258 and examined by fluorescence microscopy. The autosomes fluoresce homogenously and similarly in both species while the sex chromosomes differ in the location and brightness of some heterochromatic blocks. These cytochemical differences allow the cytotaxonomic identification of these cryptic species.
\end{abstract}

\section{INTRODUCTION}

THE Anopheles gambiae complex includes the most efficient vectors of human malaria and filariasis in Africa south of Sahara. Crossing experiments and cytogenetic studies have led to the recognition of six sibling species (merus, melas, species $A, B, C$ and $D$ ). These are characterised by a high degree of morphological crypticism and by marked biological divergences involving different levels of medical importance and different responses to insecticide treatment (Davidson, 1964; Coluzzi and Sabatini, 1967, 1968, 1969; Davidson and Hunt, 1973; White, 1974). Correct evaluation of the epidemiological parameters and of control measures requires identification of these siblings, particularly where they occur sympatrically.

In the $A$, gambiae complex, polytene chromosome studies have shown interspecific rearrangements involving paracentric inversions (Coluzzi and Sabatini, 1967, 1968, 1969; Davidson and Hunt, 1973). Alternative sequences are fixed in homozygous states for the various siblings species and constitute the taxonomic characters routinely utilised in the identification of fourth-stage larvae (salivary gland polytene chromosomes) and of bloodfed adult females (nurse cell polytene chromosomes) (Coluzzi, 1968). The mitotic chromosomes appeared substantially identical in all members of the complex with the exception of melas whose sex chromosomes are clearly differentiated by the length of their heterochromatic zone (Coluzzi and Sabatini, 1969).

Recent studies in Drosophila have shown the possibility of detecting interspecific differences in the heterochromatin with fluorescence banding techniques (Ellison and Barr, 1971; Holmquist, 1975a, b; Gatti et al., 1976). This suggested the use of the same techniques in an attempt to differentiate the species from the $A$. gambiae complex at the level of the mitotic karyotype. Species $A$ and $B$ were taken into consideration since they are the two

Postal address: Dr Sergio Pimpinelli, Istituto di Genetica, Facoltà di Scienze, Città Universitaria, 00185, Roma, Italy. 
members of the complex which are most widespread in Africa and which most frequently occur sympatrically. The fluorescence pattern obtained with the benzimidazol derivative Hoechst 33258 which binds and specifically stains AT rich DNA (Weisblum and Haenssler, 1974; Comings, 1975; Latt and Wohlleb, 1975; Gatti et al., 1976) was studied. Cytochemical differences in the heterochromatin of species $A$ and $B$ were observed with this technique, providing evidence for its usefulness at the cytotaxonomic level.

\section{Material ANd methods}

The material examined consisted of laboratory strains kindly provided by Dr G. Davidson, London School of Hygiene and Tropical Medicine. These strains are respectively from Garki, Northern Nigeria and Gezira, Sudan in the case of species $B$, and from Natsini and Garki, Northern Nigeria in the case of species $A$. The neural ganglia obtained by dissecting larvae at various stages (I, II, III and IV) or by dissecting carly pupae, were placed for 1 hour 30 minutes in saline $(0 \cdot 07$ per cent $\mathrm{NaCl})$ containing $10^{-5} \mathrm{M}$ colchicine. After hypotonic treatment they were fixed (Gatti et $a l ., 1974 a, b)$ and squashed in 45 per cent acetic acid under a siliconised coverslip. The coverslip was removed by freezing on dry ice and the slides were stained with Hoechst, mounted and studied with the methods already developed for Drosophila (Gatti et al., 1976).

\section{Results and discussion}

The karyotype of $A$. gambiae consists of two pairs of submetacentric autosomes and, in males, of telocentric $\mathrm{X}$ and $\mathrm{Y}$ chromosomes. The centromeric areas of the autosomes, the proximal half of the $\mathrm{X}$ chromosome and the whole Y chromosome, are heterochromatic (Coluzzi and Sabatini, 1967, 1969). Fig. 1 comparatively shows some $A$. gambiae $A$ and $B$ metaphases stained with Hoechst 33258. As can be seen, the autosomes fluoresce homogeneously and similarly in both species while the sex chromosomes have a species-specific fluorescence pattern. The $\mathrm{X}$ chromosome of species $A$ differs from that of species $B$ in the location and appearance of the Hoechst bright block. In addition, the $\mathrm{Y}$ chromosome of $A$ has a bright proximal area, unlike the $\mathrm{Y}$ of $B$ which is entirely fluorochrome dull. These fluorescence patterns are given idiogrammatically in fig. 2 .

From figs. 1 and 2 it is evident that the difference in the fluorescence patterns of species $A$ and $B$ cannot be simply the result of inversions. The sex chromosomes of $A$ and $B$, whatever their origin, show clear qualitative differences in their heterochromatin. Further studies on samples drawn from other populations of the two species may provide more precise indications as to the stability of the relative heterochromatin patterns and as to the existence of intraspecific polymorphism.

Striking cytochcmical differences in the heterochromatin were observed in Drosophila hydei sibling species (Holmquist, 1975a), between Drosophila melanogaster and Drosophila simulans (Ellison and Barr, 1971; Gatti et al., 1976) and in some closely related species of the Drosophila virilis group (Holmquist, 1975b; Gatti et al., 1976). The present data confirm these observations for mosquito material and strongly suggest that the qualitative and quantitative variations in the heterochromatin, perhaps like the euchro- 


\section{Plale}
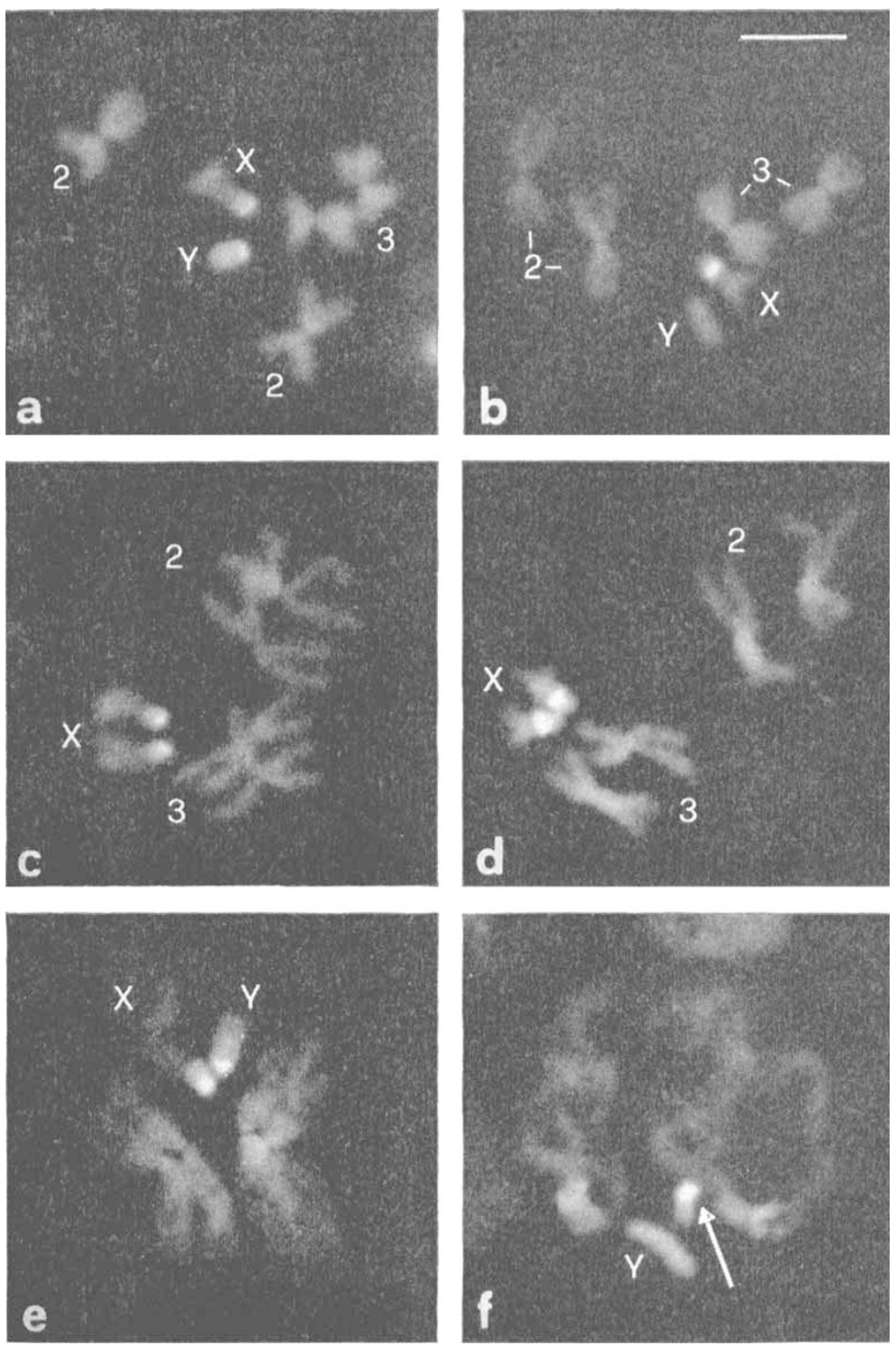

Fig. 1..... Neuroblast chromosome preparations of Anopheles gambiae species $A$ and $B$ stained with $0.05 \mu \mathrm{g} / \mathrm{ml}$ Hoechst 33258. of metaphase (a), of metaphase (c) and ot prometaphase (c) of A. gambiae A; $\delta$ metaphase (b), o metaphase (d) and $\delta$ prophase (f) of A. gambiae $B$. Note that the fluorescence pattern does not vary with condensation of the chromosomes. In (f) the arrow points to the Hoechst bright block of the X chromosome. The bar scale represents $5 \mu \mathrm{m}$. 
matic rearrangements, have played an important role in speciation. To this end we wish to point out that, because of the great number of taxonomically well-defined species they include, the sibling complexes of the genus Anopheles (such as the complexes gambiae and maculipennis) represent an ideal material for this type of study. Besides the fluorescence banding techniques, those of differential staining with Giemsa (Pimpinelli et al., 1976) and of selective decondensation of chromatin (Pimpinelli et al., 1975; Gatti et al., 1976) may also be useful in detecting any interspecific variations in the heterochromatin of Anopheles.

A. gambiae A

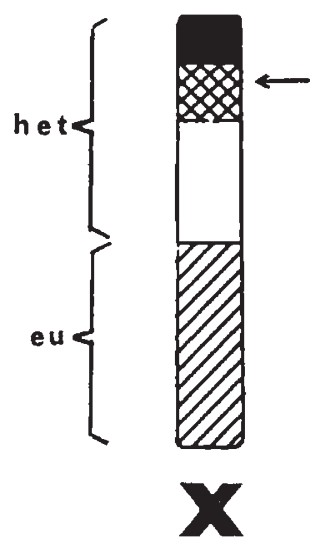

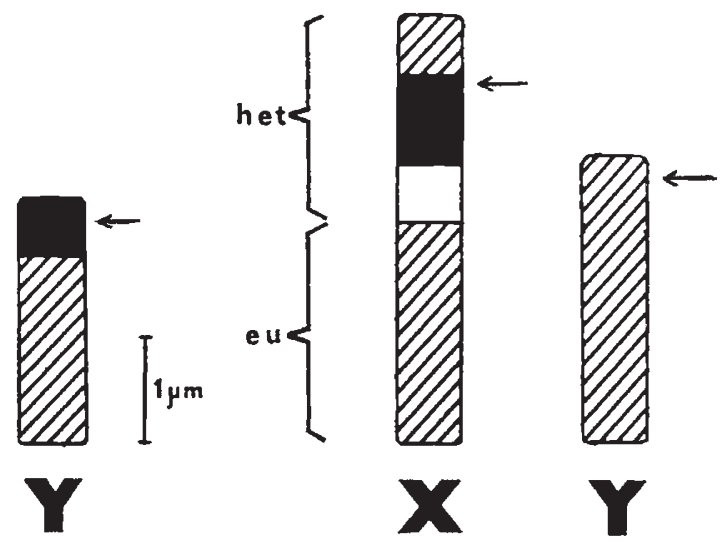

\section{A. gambiae B}

FIG. 2.-Diagrammatic representation of fluorescence pattern of sex chromosomes of Anopheles gambiae species $A$ and $B: \mathbf{\square}$, extremely bright areas; bright areas; $\square$, dull areas (with the same fluorescence as euchromatin); $\square$, areas less fluorescent than euchromatin. The arrows point to the centromere positions as inferred from the observation of some anaphase chromosomes. The idiograms were constructed by measuring the relative dimensions of the various fluorescent blocks on a number of microphotographs. For each species an equal number of contracted metaphases and prometaphases were taken into account. Therefore the idiogram is representative of lengthened metaphases of the tÿpe shown in fig. 1 (c) and (d). het: heterochromatin; eu : euchromatin.

The differences between species $A$ and $B$ shown by the $\mathrm{H}$. 33258 banding technique are likely to constitute suitable cytotaxonomic characters which can supplement those available from the polytene chromosomes, extending the species identification to all larval stages and to the pupa. It should be stressed that the technique can be easily applied to field material since the squash preparations involve only a minimum of laboratory facilities and may be preserved for long periods.

Acknowledgments:-This investigation received financial support from World Health Organisation, Geneva.

\section{REFERENGES}

cotuzzi, м. 1968. Cromosomi politenici delle cellule nutrici ovariche nel complesso gambiae del genere Anopheles. Parassitologia, 10, 179-184.

ColuzZI, M., AND SABATINI, A. 1967. Cytogenetic observations on species $A$ and $B$ of the Anopheles gambiae complex. Parassitologia, 9, 73-88. 
Coluzzi, m., And sabatini, A. 1968. Cytogenetic observations on species C of the Anopheles gambiae complex. Parassitologia, 10, 155-165.

COluzzr, M., AND SABATINI, A. 1969. Cytogenetic observations on the salt water species, Anopheles merus and Anopheles melas, of the gambiae complex. Parassitologia, 11, 177-187.

comings, D. E. 1975. Mechanisms of chromosome banding VIII. Hoechst 33258-DNA interaction. Chromosoma (Berl.), 52, 229-243.

Davidoson, G. 1964. The five mating types in the Anopheles gambiae complex. Riv. Malariol. $43,167-183$.

DAvioson, G., and HUNT, R. 1973. The crossing and chromosome characteristics of a new, sixth species in the Anopheles gambiae complex. Parassitologia, 15, 121-128.

ELLISON, J. R., AND BARR, H. J. 1971. Differences in the quinacrine staining of the chromosomes of a pair of sibling species: Drosophila melanogaster and Drosophila simulans. Chromosoma (Berl.), 34, 424-435.

GATT1, M., TANZARELLA, C., AND OLIVIERI, G. 1974a. Variation with sex of irradiation induced chromosome damage in somatic cells of Drosophila melanogaster. Nature, 247, $151-152$.

GatTi, M., TANZARElla, c., AND olivieri, G. 1974b. Analysis of the chromosome abberations induced by X-rays in somatic cells of Drosophila melanogaster. Genetics, 77, 701-719.

GATTI, M., PIMPINElli, s., AND SANTINI, G. 1976. Ciharacterization of Drosophila heterochromatin. I. Staining and decondensation with Hoechst 33258 and Quinacrine. Chromosoma (Berl.), 57, 351-375.

HolmQuist, G. 1975a. Hoechst 33258 fluorescent staining of Drosophila chromosomes. Chromosoma (Berl.), 49, 333-356.

HolmQuist, G. 1975b. Organisation and evolution of Drosophila virilis heterochromatin. Nature, 257, 503-506.

LATT, s. A., AND WOHLleb, J. C. 1975. Optical studies of the interaction of 33258 Hoechst with DNA, chromatin, and metaphase chromosomes. Chromosoma (Berl.), 52, 297-316.

pimpinelli, s., SANTINI, G., AND GATTI, M. 1976. Characterization of Drosophila heterochromatin. II. C-and N-banding. Chromosoma (Berl.), 57, 377-386.

PIMPINELl, s., GATTI, M., AND DE MARCO, A. 1975. Evidence for heterogeneity in heterochromatin of Drosophila melanogaster. Nature, 256, 335-337.

WEISBlum, B., AND HAENssler, E. 1974. Fluorometric properties of the bibenzimidazol derivative Hoechst 33258, a fluorescent probe specific for AT concentration in chromosomal DNA. Chromosoma (Berl.), 46, 255-260.

WHITE, G. B. 1974. Anopheles gambiae complex and disease transmission in Africa. Trans. R. Soc. Trop. Med. Hyg., 68, 278-301. 